International Journal of

Environmental Research and

Public Health

ISSN 1660-4601

Review

www.mdpi.com/journal/ijerph

\title{
Potential for Improved Glycemic Control with Dietary Momordica charantia in Patients with Insulin Resistance and Pre-Diabetes
}

\author{
Jimmy T. Efird ${ }^{1, \dagger, *}$, Yuk Ming Choi ${ }^{2, \dagger}$, Stephen W. Davies ${ }^{3, \dagger}$, Sanjay Mehra ${ }^{4, \dagger}$, \\ Ethan J. Anderson ${ }^{5,6, \dagger}$ and Lalage A. Katunga ${ }^{5, \dagger}$
}

1 East Carolina Heart Institute and the Center for Health Disparities, Brody School of Medicine, East Carolina University, Greenville, NC 27834, USA

2 Adult Gerontological Nurse Practitioner Program, School of Nursing, University of North Carolina at Greensboro, Greensboro, NC 27402, USA; E-Mail: y_choi6@uncg.edu

3 Department of General Surgery, University of Virginia School of Medicine, Charlottesville, VA 22908, USA; E-Mail: sd2wf@virginia.edu

4 Department of Cardiovascular Sciences, Brody School of Medicine, East Carolina University, Greenville, NC 27834, USA; E-Mail: mehras@ecu.edu

5 Department of Pharmacology and Toxicology, Brody School of Medicine, East Carolina University, Greenville, NC 27834, USA; E-Mails: andersonet@ecu.edu (E.J.A.);

katungal10@students.ecu.edu (L.A.K.)

6 East Carolina Diabetes and Obesity Institute (ECDOI) at East Carolina University, 115 Heart Drive, East Carolina Heart Institute, Greenville, NC 27834, USA

$\dagger$ These authors contributed equally to this work.

* Author to whom correspondence should be addressed; E-Mail: jimmy.efird@stanfordalumni.org; Tel.: +1-650-248-8282.

Received: 23 December 2013; in revised form: 2 February 2014 / Accepted: 10 February 2014 / Published: 21 February 2014

\begin{abstract}
Bitter Melon (Momordica charantia) is a widely used traditional remedy for hyperglycemia. While the medicinal properties of this plant have been studied extensively using in vitro and animal models, the clinical efficacy and safety in humans is largely unknown. This review discusses the benefits and limitations of bitter melon supplementation in the context of epidemic levels of insulin resistance and pre-diabetes throughout the world.
\end{abstract}


Keywords: Momordica charantia; bitter melon; insulin resistance; pre-diabetes; glycemic control

\section{Introduction}

An estimated 79 million adult Americans have pre-diabetes, a condition that is preventable and treatable if recognized early [1]. Patients presenting with pre-diabetes have glycated hemoglobin (HgAlc) and blood sugar levels that are below the clinical threshold to be classified as type 2 diabetes but are higher than normal (HgAlc 5.7-6.4\%; fasting blood glucose 100-125 mg/dL; 2-hour plasma glucose with $75 \mathrm{~g}$ oral glucose tolerance test between 140-199) [2]. However, the early stages of damage to the heart and circulatory system have already begun and without intervention, pre-diabetics are at risk for becoming diabetic and developing cardiovascular disease [3,4]. While the percentage of pre-diabetes is equal among blacks and whites (35\%), a disproportionate percentage of non-Hispanic blacks progress to diabetes compared with their non-Hispanic white counterparts (18.7\% vs. 10.2\%) [1]. Guidelines established by the American Association of Clinical Endrocrinologists (AACE) suggest that regular glucose monitoring and adoption of healthful lifestyles (diet and exercise) may reverse uncomplicated pre-diabetes [5].

Nearly $40 \%$ of Americans use complementary and alternative medicine (CAM) for health promotion and the treatment of illness [6]. Among this group, between 2 and 3 million specifically use CAM in an attempt to lower blood sugar levels and to treat various stages of diabetes, despite limited studies of their safety and efficacy [7]. CAM is most commonly used by immigrant groups and their descendants, including Asians, Latin Americans, Africans, Indians, West Indians, and Native Americans [7]. However, CAM use is not limited to these groups and many Americans have become increasingly aware of CAM practices from their immigrant and Native American neighbors. Because of the rapidly changing demographics of the United States and the wide use of CAM, primary care providers need to be knowledgeable about CAM in order to appropriately advise their patients regarding the safety and efficacy of these practices. For example, potentially harmful interactions with conventional medications may occur if an effective CAM therapy is added to conventional therapy [7]. Furthermore, some patients, especially among vulnerable populations, may not disclose CAM use to their primary care provider because of historic mistrust of the health care system [8]. Many CAM therapies are based on traditional remedies and have not been evaluated under rigorous scientific conditions (e.g., largescale, placebo-controlled, randomized clinical trials using standardized protocols) [7]. Nonetheless, $25 \%$ of prescription drugs on the market today contain active compounds derived from plants or plantderived synthetic analogs (e.g., metformin and the French lilac plant) [9]. Approximately $80 \%$ of the world's population routinely depends on plant derived therapies to prevent and treat illness [9].

Previous animal studies have observed an increase in insulin secretion and glucose uptake with the use of some herbal compounds [10]. However, it remains important to carefully evaluate the potential effectiveness and safety of these compounds in controlling blood sugar levels in humans. Evidence of the therapeutic benefits of folkloric medications cannot be presumed solely from their historical use or data extracted from animal studies alone. Below, we review the clinical evidence to date pertaining to 
Momordica charantia (bitter melon), a traditional herbal remedy used since ancient times by Chinese and Ayurvedic practitioners to treat high blood sugar and early signs of diabetes $[11,12]$.

\section{Description}

Bitter melon is a commonly consumed vegetable that is found throughout the sub-tropical world (China, India, Thailand, East Africa, The Caribbean, Central and South America) and is known by various names, such as balsam pear, bitter gourd, carilla, cerasee (wild variety), cundeamor, goo-fah, and karela [13,14]. The plant has been well studied in animal models and several mechanisms of action have been proposed for its anti-diabetic properties (Table 1). At latest count, approximately 228 different compounds with possible medicinal properties, acting alone or in combination, have been isolated from bitter melon fruit, seeds, leaves, stems, pericaps, endosperm, callus tissues, and cotyledons [15]. Among these, the most actively studied constituents shown to improve glycemic control include charatin, polypeptide-p, vicine, momordin, and similar derivatives (e.g., momordinol, momordicilin, momorcharin, momordicin) [15-17].

Table 1. Antidiabetic mechanisms of bitter melon (Momordica charantia) in animal models.

\begin{tabular}{|c|c|c|}
\hline Mode of Action & Model & Observed Effects \\
\hline \multirow[t]{4}{*}{$\begin{array}{l}\text { 1. Insulin signaling } \\
\text { pathway }\end{array}$} & $\begin{array}{l}\text { - } \quad d b / d b \text { mice } \\
\text { - } \quad \text { Whole fruit powder, lipid, } \\
\text { saponin or the } \\
\text { hydrophilic residue of } \\
\text { bitter melon fruit } \\
\text { - } 5 \text { weeks }\end{array}$ & $\begin{array}{l}\text { - } \quad \downarrow \text { Hbalc } 10 \% \\
\text { - } \quad \downarrow \text { Protein tyrosine phosphatase 1B (PTP 1B) activity } \\
\text { by } 20 \%[18]\end{array}$ \\
\hline & $\begin{array}{l}\text { - } \quad \text { Non- obese diabetic mice } \\
\text { (active ingredient isolated } \\
\text { from Bitter Melon) } \\
\end{array}$ & - $\quad \downarrow$ Glucose after $8 \mathrm{~h}$ intraperitoneal injection [19] \\
\hline & $\begin{array}{l}\text { - } \text { C57B16/J mice, high fat } \\
\text { diet } \\
\text { - } \text { Bitter melon fruit } \\
\text { - } 16 \text { weeks }\end{array}$ & $\begin{array}{l}\text { - } \quad \uparrow \text { Liver tyrosine phosphorylation } \\
\text { - } \quad \uparrow \text { Insulin receptor substrate } 1 \\
\text { - } \quad \downarrow \text { Plasma apoB-100 and apoB-48 in HFD-fed mice } \\
\quad[20]\end{array}$ \\
\hline & $\begin{array}{l}\text { - C57B16/J mice, high fat } \\
\text { diet } \\
\text { - } \text { Bitter melon, aquatic } \\
\text { extract powder } \\
\text { - } 12 \text { weeks }\end{array}$ & $\begin{array}{l}\text { In skeletal muscle: } \\
\text { - } \quad \uparrow \text { Insulin receptor substrate-1 (IRS-2) } \\
\text { - } \quad \uparrow \text { Insulin receptor } \beta \text { (IR } \beta \text { ) } \\
\text { - } \quad \uparrow \text { Phosphatidylinositide 3-kinases (PI 3-K) } \\
\text { - } \quad \uparrow \text { Glucose transporter type 4 (GLUT4) } \\
\text { - } \quad \uparrow \text { Phosphorylation of insulin receptor substrate-1, } \\
\quad \text { Akt1 and Akt2 } \\
\text { - } \quad \downarrow \text { Body weight, plasma glucose, insulin, leptin levels, } \\
\quad \text { and HOMA-IR [21] }\end{array}$ \\
\hline
\end{tabular}


Table 1. Cont.

\begin{tabular}{|c|c|c|c|}
\hline & Mode of Action & Model & Observed Effects \\
\hline & \multirow[t]{3}{*}{$\begin{array}{l}\text { Gluconeogenic \& } \\
\text { Glycolysis enzymes }\end{array}$} & $\begin{array}{l}\text { - } \text { Rats } \\
\text { - } \text { Bitter melon fruits } \\
\text { extracts }\end{array}$ & $\begin{array}{l}\text { - } \quad \downarrow \text { Hexokinase activity and glucose uptake activity in } \\
\text { intestinal fragments [22] }\end{array}$ \\
\hline & & $\begin{array}{l}\text { - } \text { Streptozotocin treated rats } \\
\text { - } \quad \text { Bitter melon leaf extract } \\
\text { - } 90 \mathrm{~min} \\
\end{array}$ & $\begin{array}{l}\text { - } \quad \downarrow \text { Activity } \sim 30 \% \text { Hepatic glucose-6-phosphatase and } \\
\text { fructose 1,6-bisphosphatase activities [23] }\end{array}$ \\
\hline & & $\begin{array}{l}\text { - } \text { C57B16/J, high fat diet } \\
\text { - } \quad \text { Bitter melon fruit extract } \\
\text { - } 4 \text { weeks }\end{array}$ & $\begin{array}{ll}\text { - } & \uparrow \text { mRNA and protein expression of glucose } \\
\text { - } & \downarrow \text { Hyperglycemia, hyperleptinemia, HbAlc and free } \\
& \text { fatty acid } \\
\text { - } & \uparrow \text { Adipose PPAR } \gamma \text { and liver PPAR } \gamma \text { mRNA levels } \\
& {[24]}\end{array}$ \\
\hline & $\begin{array}{l}\text { Pancreatic Beta } \\
\text { cells }\end{array}$ & $\begin{array}{l}\text { - } \text { Streptozotocin Wistar rats } \\
\text { - } \text { Bitter melon fruit pulp } \\
\text { - } 28 \text { days }\end{array}$ & $\begin{array}{l}\text { - } \quad \uparrow \text { Fasting blood, glucose, serum insulin and cell } \\
\text { function } \\
\text { - } \quad \uparrow \text { Diabetic rat cells were abundant with insulin } \\
\text { granules } \\
\text { - } \quad \uparrow \text { Islet size and total cell area } \\
\text { - } \quad \text { Number of } \beta \text {-cells } \uparrow 2 \text { fold with bitter melon treatment } \\
\quad[25]\end{array}$ \\
\hline & & $\begin{array}{l}\text { - Alloxan treated albino } \\
\text { rats } \\
\text { - } \quad \text { Bitter melon fruit } \\
\text { - } 15 \text { and } 30 \text { days }\end{array}$ & 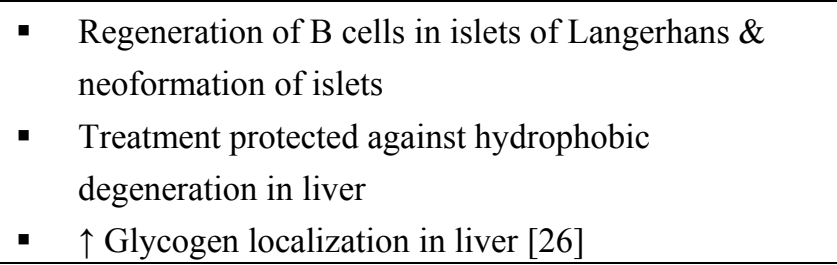 \\
\hline & \multirow[t]{3}{*}{ Lipid Regulation } & $\begin{array}{ll}\text { - } & \text { C57bl6/J, high fat diet } \\
\text { - } & \text { Bitter melon seed oil } \\
\text { - } & 5 \text { weeks }\end{array}$ & $\begin{array}{l}\text { White adipose tissue: } \\
\text { - } \quad \uparrow \text { Phosphorylation of acetyl-CoA carboxylase } \\
\text { - } \quad \uparrow \text { cAMP-activated protein kinase (PKA) [27] }\end{array}$ \\
\hline & & $\begin{array}{l}\text { - } \mathrm{C} 57 \mathrm{BL} / 6 \text {, high fat diet } \\
\text { - } \quad \text { Bitter melon fruit } \\
\text { - } 12 \text { weeks }\end{array}$ & $\begin{array}{l}\text { Epididymal adipose tissues (EAT) and brown adipose } \\
\text { tissues (BAT) } \\
\text { - } \quad \downarrow \text { Mast cell recruitment } \\
\text { - } \quad \downarrow \text { Interleukin-6 (IL-6) and tumor necrosis factor- } \alpha \\
\quad(\text { TNF- } \alpha) \\
\text { - } \quad \downarrow \text { Macrophage infiltration } \\
\text { - } \quad \downarrow \text { M1/M2 phenotype ratio of macrophages [28] }\end{array}$ \\
\hline & & $\begin{array}{l}\text { - } \quad \text { Sprague-Dawley rats, } \\
\text { high fat diet } \\
\text { - } \quad \text { Bitter melon juice } \\
\text { - } 7 \text { weeks }\end{array}$ & $\begin{array}{ll}\text { - } & \downarrow \text { Visceral fat mass, hepatic triacylglycerol } \\
\text { - } & \uparrow \text { Serum free fatty acids } \\
\text { - } & \uparrow \text { Peroxisome proliferator-activated receptor } \\
& \text { coactivator-1 } \alpha \text { (PGC-1- } \alpha)[29]\end{array}$ \\
\hline
\end{tabular}

\section{Clinical Reports}

The clinical potential of bitter melon has been examined in several human cell line experiments. In studies of hepatoblastoma cells (HepG2), it has been shown to be a potent inhibitor of triglyceride synthesis and ApoB secretion [30]. Similarly, momordin (a constituent of bitter melon) increases the 
expression of peroxisome proliferator-activated receptor (PPAR) $\delta$ mRNA in HepG2, which is important in the regulation of glucose metabolism and fatty acid storage [31]. Bitter melon juice also has been demonstrated to significantly reduce sterol regulatory element-binding protein 1c (SREBP-1c) when applied to primary preadipocytes, further highlighting the prospective benefit of this compound in glycemic control. However, direct evidence in humans has been limited to mostly anecdotal case reports and a few conflicting studies among diabetic or non-diabetic "at-risk" patients (Table 2). Accordingly, the effect of bitter melon on blood sugar levels and disease progression in pre-diabetics must be extrapolated from these studies.

Table 2. Summary of clinical studies on bitter melon (Momordica charantia) ${ }^{\S}$.

\begin{tabular}{|c|c|c|c|c|c|}
\hline $\begin{array}{l}\text { First Author } \\
\text { [Ref.] } \\
\text { (Location) }\end{array}$ & Sample & Design & Treatment & $\begin{array}{l}\text { Jadad } \\
\text { Score }\end{array}$ & Results \\
\hline $\begin{array}{l}\text { Ahmad [32] } \\
\text { (Bangladesh) }\end{array}$ & $\begin{array}{l}100 \text { Moderate type II } \\
\text { diabetics }\end{array}$ & $\begin{array}{l}\text { Case series } \\
\text { (no referent group) }\end{array}$ & $\begin{array}{l}\text { Aqueous homogenized } \\
\text { suspension (single treatment), } \\
\text { Non-standardized dose }\end{array}$ & $\mathrm{C}$ & $\begin{array}{l}\downarrow \text { Fasting serum } \\
\text { glucose } \\
(p<0.001), \\
\downarrow \text { Post-prandial } \\
\text { serum glucose } \\
(p<0.001)\end{array}$ \\
\hline $\begin{array}{l}\text { Akhtar [33] } \\
\text { (Pakistan) }\end{array}$ & $\begin{array}{l}8 \text { Uncomplicated, } \\
\text { maturity-onset } \\
\text { diabetics }\end{array}$ & $\begin{array}{l}\text { Case series } \\
\text { (no referent group) }\end{array}$ & $\begin{array}{l}\text { Powdered bitter melon, } \\
50 \mathrm{mg} / \mathrm{kg} \text { body weight twice } \\
\text { daily for } 7 \text { days after breakfast } \\
\text { and dinner along with milk }\end{array}$ & $\mathrm{C}$ & $\begin{array}{l}\downarrow \text { Mean blood } \\
\text { sugar levels } \\
(p<0.05)\end{array}$ \\
\hline $\begin{array}{l}\text { Baldwa [34] } \\
\text { (India) }\end{array}$ & $\begin{array}{l}5 \text { Healthy } \\
\text { volunteers, } \\
5 \text { Diabetic referents, } \\
9 \text { Pts. with diabetes } \\
\text { mellitus }\end{array}$ & $\begin{array}{l}\text { Unblinded, } \\
\text { Non-randomized, } \\
\text { Clinical trial }\end{array}$ & $\begin{array}{l}\text { Subcutaneous (single injection) } \\
\text { of Polypepide-p, } \\
\text { Arbitrary dosing }\end{array}$ & $\mathrm{C}$ & $\begin{array}{l}\downarrow \text { Fasting serum } \\
\text { glucose } \\
(p<0.05)\end{array}$ \\
\hline $\begin{array}{l}\text { Dans [35] } \\
\text { (Philippines) }\end{array}$ & $\begin{array}{l}40 \text { Newly physician } \\
\text { diagnosed or poorly } \\
\text { controlled type } 2 \\
\text { diabetes }\end{array}$ & $\begin{array}{l}\text { Double-blind, } \\
\text { Randomized, } \\
\text { Placebo- } \\
\text { controlled, } \\
\text { Intent-to-treat }\end{array}$ & $\begin{array}{l}2 \text { Capsules of bitter melon thrice } \\
\text { daily for } 3 \text { months, } \\
\text { Medication prepared by } 3 \text { rd } \\
\text { party to conceal allocation }\end{array}$ & B & $\begin{array}{l}\downarrow \text { Fasting plasma } \\
\text { glucose } \\
(p=0.5862), \\
\text { Achieved study } \\
\text { power was only } \\
11 \%\end{array}$ \\
\hline $\begin{array}{l}\text { Fuangchan } \\
\text { [36] } \\
\text { (Thailand) }\end{array}$ & $\begin{array}{l}143 \text { Newly } \\
\text { diagnosed type } 2 \\
\text { diabetes }\end{array}$ & $\begin{array}{l}\text { Double-blind, } \\
\text { Active-control, } \\
\text { Pill counting to } \\
\text { monitor } \\
\text { compliance, } \\
\text { Intent-to-treat }\end{array}$ & $\begin{array}{l}2 \text { Capsules }(500 \mathrm{mg} \text { ) before and } \\
\text { after meals for } 4 \text { weeks }\end{array}$ & A & $\begin{array}{l}\downarrow \text { Mean } \\
\text { fructosamine } \\
\text { levels } \\
(-10.2 \mu \mathrm{mol} / \mathrm{L} ; \\
95 \% \mathrm{CI}=-19.1 \\
\text { to }-1.3)\end{array}$ \\
\hline $\begin{array}{l}\text { Grover [37] } \\
\text { (India) }\end{array}$ & $\begin{array}{l}14 \text { NIDDM } \\
\text { (mixed sex), } \\
6 \text { IDDM } \\
\text { (female only) }\end{array}$ & $\begin{array}{l}\text { Case series (no } \\
\text { referent group) }\end{array}$ & $\begin{array}{l}\text { Bitter melon seeds, } \\
\text { Single treatment }\end{array}$ & $\mathrm{C}$ & $\begin{array}{l}\downarrow \text { Post-prandial } \\
\text { serum glucose } \\
(p=0.001)\end{array}$ \\
\hline
\end{tabular}


Table 2. Cont.

\begin{tabular}{|c|c|c|c|c|c|}
\hline $\begin{array}{l}\text { First Author } \\
\text { [Ref.] } \\
\text { (Location) } \\
\end{array}$ & Sample & Design & Treatment & $\begin{array}{l}\text { Jadad } \\
\text { Score }\end{array}$ & Results \\
\hline $\begin{array}{l}\text { Habib [38] } \\
\text { (Bangladesh) }\end{array}$ & $\begin{array}{l}8 \mathrm{NIDDM}(3 \mathrm{~F}, 5 \mathrm{M}) \\
\text { taking } \\
1 / 2-1 \text { tablet } \\
\text { glibenclamide } \\
\text { randomly selected } \\
\text { from diabetic center }\end{array}$ & $\begin{array}{l}\text { Open-label, Cross- } \\
\text { over design ( } 15 \text { day } \\
\text { wash-out period } \\
\text { between treatments) }\end{array}$ & $\begin{array}{l}\text { Powdered, dried fruit } \\
\text { ( } 4 \text { gm/patient) administered } \\
\text { consecutively for } \\
21 \text { days }\end{array}$ & $\mathrm{C}$ & $\begin{array}{l}\downarrow \text { Post- } \\
\text { prandial } \\
\text { serum } \\
\text { glucose } \\
(p=0.001)\end{array}$ \\
\hline $\begin{array}{l}\text { John [39] } \\
\text { (India) }\end{array}$ & 26 Type 2 diabetics & $\begin{array}{l}\text { Unblinded, } \\
\text { Randomized, Clinical } \\
\text { trial }\end{array}$ & $\begin{array}{l}2 \text { Tablets }(1 \mathrm{gm}) \text { dried fruit } \\
\text { thrice daily after meals for } 4 \\
\text { weeks }\end{array}$ & $\mathrm{C}$ & $\begin{array}{l}\downarrow \text { Fasting } \\
\text { serum } \\
\text { glucose } \\
(p>0.05), \\
\downarrow \text { Post- } \\
\text { prandial } \\
\text { serum } \\
\text { glucose } \\
(p>0.05)\end{array}$ \\
\hline $\begin{array}{l}\text { Kasbia [14] } \\
\text { (Canada) }\end{array}$ & $\begin{array}{l}5 \text { Non-diabetic, } \\
\text { overweight men }\end{array}$ & $\begin{array}{l}\text { Randomized, } \\
\text { Blinded, } \\
\text { Placebo-controlled, } \\
\text { Cross-over study }\end{array}$ & $\begin{array}{l}\text { Eligible participants } \\
\text { underwent } 3 \text { experimental } \\
\text { conditions in a randomized } \\
\text { order: placebo, } \\
50 \mathrm{mg} / \mathrm{kg}, 100 \mathrm{mg} / \mathrm{kg} \text { body } \\
\text { weight of freeze-dried juice, } \\
\text { Single treatments separated } \\
\text { by } 1 \text {-week washout period, } \\
\text { Placebo capsules filled with } \\
\text { inert cellulose }\end{array}$ & $\mathrm{C}$ & $\begin{array}{l}\text { No effect on } \\
\text { plasma } \\
\text { glucose }\end{array}$ \\
\hline $\begin{array}{l}\text { Khanna [40] } \\
\text { (India) }\end{array}$ & $\begin{array}{l}6 \text { Juvenile referents, } \\
5 \text { Juvenile diabetes, } \\
2 \text { Maturity onset } \\
\text { diabetes referents, } \\
6 \text { Maturity onset } \\
\text { diabetes }\end{array}$ & $\begin{array}{l}\text { Unblinded, } \\
\text { Non-randomized, } \\
\text { Clinical trial }\end{array}$ & $\begin{array}{l}\text { Subcutaneous, } \\
\text { Polypepide-p (single } \\
\text { treatment), } \\
\text { Arbitrary dosing depending } \\
\text { upon severity of diabetes }\end{array}$ & $\mathrm{C}$ & $\begin{array}{l}\downarrow \text { Serum } \\
\text { glucose } \\
(p<0.05) \text { at } \\
\text { selected time } \\
\text { point }\end{array}$ \\
\hline
\end{tabular}


Table 2. Cont.

\begin{tabular}{|c|c|c|c|c|c|}
\hline $\begin{array}{l}\text { First Author } \\
\text { [Ref.] } \\
\text { (Location) }\end{array}$ & Sample & Design & Treatment & $\begin{array}{l}\text { Jadad } \\
\text { Score }\end{array}$ & Results \\
\hline $\begin{array}{l}\text { Kochhar [41] } \\
\text { (India) }\end{array}$ & $\begin{array}{l}60 \text { Non- } \\
\text { insulin- } \\
\text { dependent } \\
\text { male diabetics }\end{array}$ & $\begin{array}{l}\text { Case series (no } \\
\text { referent group) }\end{array}$ & $\begin{array}{l}\text { Raw powered mixture (bitter } \\
\text { melon fruit, fenugreek seeds, } \\
\text { jambu seeds) in the form of } \\
\text { capsules (Group I) or salty } \\
\text { biscuits (Group 2), } \\
\text { Daily supplementation } \\
\text { of } 1 \mathrm{~g} \text { for } 1.5 \text { months followed } \\
\text { by } 2 \mathrm{~g} \text { for } 1.5 \text { months, } \\
\text { Fresh, immature bitter melon } \\
\text { fruit procured from a single lot } \\
\text { grown locally and stored in } \\
\text { airtight plastic containers until } \\
\text { use }\end{array}$ & $\mathrm{C}$ & $\begin{array}{l}\downarrow \text { Fasting glucose } \\
(p<0.01), \\
\downarrow \text { Postprandial glucose } \\
(p<0.01), \\
\downarrow \text { Decreased oral } \\
\text { hypoglycemic drug } \\
\text { intake }(p<0.05)\end{array}$ \\
\hline $\begin{array}{l}\text { Leatherdale } \\
\text { [42] } \\
\text { (England) }\end{array}$ & 9 NIDDM & $\begin{array}{l}\text { Case series (no } \\
\text { referent group) }\end{array}$ & $\begin{array}{l}\text { Water-soluble extract of bitter } \\
\text { melon fruit (single treatment), } \\
\text { Later received fried bitter } \\
\text { melon fruit for } 8 \text { to } 11 \text { weeks }\end{array}$ & $\mathrm{C}$ & $\begin{array}{l}\downarrow \text { Blood glucose } \\
\text { concentration during } \\
50 \text { g oral glucose } \\
\text { tolerance test } \\
(p<0.05)\end{array}$ \\
\hline $\begin{array}{l}\text { Lim [43] } \\
\text { (Philippines) }\end{array}$ & $\begin{array}{l}40 \text { Newly } \\
\text { diagnosed type } \\
2 \text { diabetes } \\
\text { mellitus ( } 18 \\
\text { males, } 22 \\
\text { females) }\end{array}$ & $\begin{array}{l}\text { Double-blind, } \\
\text { placebo- } \\
\text { controlled trial, } \\
\text { Medications } \\
\text { prepared by a } \\
\text { third party to } \\
\text { conceal allocation }\end{array}$ & $\begin{array}{l}\text { Tablets containing dried bitter } \\
\text { melon leaves, } \\
\text { Single oral dose ( } 3 \text { treatment } \\
\text { groups of } 60,80 \text {, } \\
100 \mathrm{mg} / \mathrm{kg} / \text { day), } \\
\text { Placebo tablets matched in } \\
\text { appearance to active } \\
\text { compound }\end{array}$ & $\begin{array}{l}\mathrm{B} / \mathrm{A} \\
\text { (published } \\
\text { in journal } \\
\text { without } \\
\text { impact } \\
\text { factor or } \\
\text { indexed in } \\
\text { PubMed) }\end{array}$ & $\begin{array}{l}\uparrow \text { Insulin levels at } \\
15 \text { min ( } p=0.0402) \text {, } \\
\downarrow \text { Average plasma } \\
\text { glucose levels at } \\
15 \mathrm{~min} \text { ( } p=0.0121) \text {, } \\
100 \mathrm{mg} / \mathrm{kg} / \text { day dose } \\
\text { of bitter melon was } \\
\text { more effective than } \\
\text { lower doses and } \\
\text { placebo in reducing } \\
\text { mealtime glycemic } \\
\text { excursions within } 4 \\
\text { hour postdose, with } \\
\text { more rapid return to } \\
\text { baseline levels }\end{array}$ \\
\hline
\end{tabular}


Table 2. Cont.

\begin{tabular}{|c|c|c|c|c|c|}
\hline $\begin{array}{l}\text { First Author } \\
\text { [Ref.] } \\
\text { (Location) }\end{array}$ & Sample & Design & Treatment & $\begin{array}{l}\text { Jadad } \\
\text { Score }\end{array}$ & Results \\
\hline $\begin{array}{l}\text { Pons [44] } \\
\text { (Puerto Rico) }\end{array}$ & $\begin{array}{l}8 \text { Patients } \\
(7 \text { diabetic, } \\
1 \text { normal) }\end{array}$ & $\begin{array}{l}\text { Case series (no } \\
\text { referent group) }\end{array}$ & $\begin{array}{l}2 \text { Pills }(0.10 \mathrm{~g} \text { of bitter } \\
\text { melon extract powder }) \\
\text { taken after each meal, } \\
\text { Duration of } \\
\text { administration varied up } \\
\text { to } 3 \text { weeks }\end{array}$ & $\mathrm{C}$ & $\begin{array}{l}\downarrow \text { Blood sugar in } 2 \\
\text { patients }(p>0.05)\end{array}$ \\
\hline $\begin{array}{l}\text { Purification, } \\
\text { (unpublished } \\
\text { report in Duque } \\
\text { [45]) } \\
\text { (Phillipines) }\end{array}$ & $\begin{array}{l}260 \text { Type } 2 \\
\text { diabetics }\end{array}$ & $\begin{array}{l}\text { Phase III trial, } \\
\text { Randomized block } \\
\text { design, } \\
\text { (bitter melon, } \\
\mathrm{n}=128 ; \\
\text { glibenclamide, } \\
\mathrm{n}=132 \text { ) }\end{array}$ & $\begin{array}{l}\text { Tablet }(100 \mathrm{mg} / \mathrm{kg} / \text { day }) \\
\text { for } 12 \text { weeks }\end{array}$ & $\begin{array}{l}\mathrm{C} \\
\text { (sufficient } \\
\text { details not } \\
\text { provided) }\end{array}$ & $\begin{array}{l}\downarrow \text { Fasting plasma } \\
\text { glucose }(p<0.05) \text {, } \\
\text { Bitter melon and } \\
\text { glibenclamide were } \\
\text { comparable in terms of } \\
\text { efficacy and safety }\end{array}$ \\
\hline $\begin{array}{l}\text { Rosales [46] } \\
\text { (Philippines) }\end{array}$ & $\begin{array}{l}27 \text { Type } 2 \\
\text { diabetics with } \\
\text { suboptimal } \\
\text { glycemic } \\
\text { control }\end{array}$ & $\begin{array}{l}\text { Unblinded, } \\
\text { Randomized, } \\
\text { Cross-over, } \\
\text { Clinical trial }\end{array}$ & $\begin{array}{l}200 \mathrm{~mL} \text { bitter melon tea } \\
\text { after meals, } \\
\text { Placebo consisted of tea } \\
\text { made from leaves of } \\
\text { Camellia sinensis, } \\
24 \text { Week study }\end{array}$ & $\mathrm{C}$ & $\begin{array}{l}\downarrow H \mathrm{HAA} 1 \mathrm{c}(63 \% \\
\text { reduction, } \\
p=0.005), \\
\downarrow \text { Fasting serum } \\
\text { glucose }(p=0.403)\end{array}$ \\
\hline $\begin{array}{l}\text { Srivastava [47] } \\
\text { (India) }\end{array}$ & $\begin{array}{l}7 \text { Mild to } \\
\text { severe } \\
\text { diabetics }\end{array}$ & $\begin{array}{l}\text { Case series (no } \\
\text { referent group) }\end{array}$ & $\begin{array}{l}100 \mathrm{~mL} \text { Aqueous } \\
\text { extract, } \\
\text { Separate group given } 15 \\
\mathrm{~g} \text { dried fruit powder } \\
\text { thrice a day in equal } \\
\text { doses of } 5 \mathrm{~g} \text { each, } \\
3 \text { Week treatment period }\end{array}$ & $\mathrm{C}$ & $\begin{array}{l}\downarrow \text { Post-prandial serum } \\
\text { glucose for aqueous } \\
\text { group only }(p<0.01)\end{array}$ \\
\hline $\begin{array}{l}\text { Tongia [48] } \\
\text { (India) }\end{array}$ & 15 NIDDM & $\begin{array}{l}\text { Unblinded, } \\
\text { Non-random } \\
\text { allocation }\end{array}$ & $\begin{array}{l}\text { 200mg Twice daily soft } \\
\text { bitter melon extract } \\
\text { ( } 7 \text { days treatment), }\end{array}$ & $\mathrm{C}$ & $\begin{array}{l}\downarrow \text { Post-prandial serum } \\
\text { glucose } \\
(p<0.001)\end{array}$ \\
\hline $\begin{array}{l}\text { Tsai [49] } \\
\text { (Taiwan) }\end{array}$ & $\begin{array}{l}42 \text { Patients } \\
\text { with metabolic } \\
\text { syndrome } \\
\text { (mean age } \\
45.7 \text {; range } 23 \\
\text { to } 63 \text { years) }\end{array}$ & $\begin{array}{l}\text { Open-label, } \\
\text { Uncontrolled, } \\
\text { Supplementation } \\
\text { trial }\end{array}$ & $\begin{array}{l}4.8 \mathrm{~g} \text { Lyophilized bitter } \\
\text { melon powder daily for } \\
3 \text { months }\end{array}$ & $\mathrm{C}$ & $\begin{array}{l}\downarrow \text { Metabolic syndrome } \\
(p=0.021) \text {, } \\
\text { Improved insulin } \\
\text { resistance at visit } 2 \\
\text { and return to nearly } \\
\text { baseline levels after } \\
\text { stopping bitter melon } \\
(p>0.05)\end{array}$ \\
\hline
\end{tabular}


Table 2. Cont.

\begin{tabular}{|c|c|c|c|c|c|}
\hline $\begin{array}{l}\text { First Author } \\
\text { [Ref.] } \\
\text { (Location) }\end{array}$ & Sample & Design & Treatment & Jadad Score & Results \\
\hline $\begin{array}{l}\text { Welhinda [50] } \\
\text { (Sri Lanka) }\end{array}$ & $\begin{array}{l}18 \text { Newly } \\
\text { diagnosed } \\
\text { maturity onset } \\
\text { diabetics }\end{array}$ & Cross-over design & $\begin{array}{l}100 \mathrm{~mL} \text { Aliquots of clear } \\
\text { bitter melon juice given } \\
\text { orally, } \\
\text { Placebo (same patients) } \\
\text { received } 100 \mathrm{~mL} \text { distilled } \\
\text { water, } \\
\text { Single administration of } \\
\text { treatment and placebo }\end{array}$ & $\mathrm{C}$ & $\begin{array}{l}\downarrow \text { Glucose } \\
\text { tolerance curves } \\
\text { compared with } \\
\text { referent glucose } \\
\text { tolerance curves } \\
(p<0.01)\end{array}$ \\
\hline $\begin{array}{l}\text { Zänker [51] } \\
\text { (Germany) }\end{array}$ & $\begin{array}{l}124 \text { Type } 2 \\
\text { diabetes } \\
\text { mellitus (age }> \\
40 \text { years) not } \\
\text { treated with } \\
\text { insulin but } \\
\text { receiving } \\
\text { anti-diabetics }\end{array}$ & $\begin{array}{l}\text { Prospective, } \\
\text { placebo- } \\
\text { controlled, } \\
\text { randomized, } \\
\text { double-blinded, } \\
\text { Participants } \\
\text { required to stop } \\
\text { taking dietary } \\
\text { supplements, } \\
\text { Compliance not } \\
\text { monitored }\end{array}$ & $\begin{array}{l}\text { Special water soluble bitter } \\
\text { melon standardized at } 10 \% \\
\text { charantin, } \\
\text { Daily dose - } 1 \mathrm{~g} \\
\text { ( } 2 \text { capsules), } \\
\text { Second active group } \\
\text { received chromium and zinc } \\
\text { supplementation, } \\
\text { Screening phase }+4 \text {-week } \\
\text { depletion phase }+4 \text {-month } \\
\text { intervention phase, } \\
\text { No information provided on } \\
\text { the country of origin for } \\
\text { bitter melon, part of the } \\
\text { plant used (e.g., seeds, pulp, } \\
\text { leaves), or self-life }\end{array}$ & $\begin{array}{l}\text { B/A } \\
\text { (published in } \\
\text { journal } \\
\text { without } \\
\text { impact factor } \\
\text { or indexed in } \\
\text { PubMed) }\end{array}$ & $\begin{array}{l}\downarrow \text { HbAlc, } \\
p=0.0002, \\
\text { No hypoglycemic } \\
\text { or other adverse } \\
\text { events reported }\end{array}$ \\
\hline
\end{tabular}

$\S$ Studies identified through a search of PubMed, Google Scholar, and published manuscript reference lists using key words (Momordica charantia, bittermelon, bitter gourd, and karela). $\mathrm{A} \geq 4, \mathrm{~B}=2-3, \mathrm{C} \leq 1 ; \mathrm{NIDDM}=$ non-insulin dependent diabetes mellitus; IDDM $=$ insulin dependent diabetes mellitus.

Studies illustrating the benefit of bitter melon on lowering blood sugar have appeared sporadically in the literature for over 70 years. On the island countries of Puerto Rico, Cuba, and the Dominican Republic, bitter melon has been traditionally used to treat high blood sugar and diabetes since the early 1900s [44]. In a non-randomized administration of bitter melon in the form of a dried powder alcoholic extract to eight patients (seven diabetic, one normal) at a clinic in San Juan, Puerto Rico, a positive blood sugar lowering effect was observed in two of the patients, one of whom was non-diabetic [44]. However, it was emphasized in this report that "the continued and indiscriminate use of any such remedy by the public, if ineffective, unavoidably results in detrimental and even fatal procrastination of appropriate treatments."

In Britain, immigrant herbal practitioners have long claimed that bitter melon (karela) is an effective therapy for lowering blood sugar levels [52]. In one of the first case reports to be published in a main-stream medical journal, bitter melon reportedly reduced the urine sugar levels of a 40 year-old 
diabetic Pakistani woman, who hitherto had difficulty controlling her diabetes with conventional drug (chlorpropamide) therapy and dietary restriction [52]. Furthermore, she was able to effectively reduce the dose of chlorpropamide necessary to control her high blood sugar. However, physicians were cautioned to be aware of this potential food-drug interaction among their Indian and Pakistani diabetic patients who also may be receiving concurrent treatment from an herbal practitioner.

Polypeptide-p is one of the few active compounds in bitter melon that has been studied in clinical trials. The extraction of polypeptide-p (consisting of 166 residues) from the fruit, seeds, and tissue of frozen bitter melon was first described at the Third International Congress of Plant Tissue and Cell Culture held in Leicester, England during the summer of 1974 [34]. The compound closely resembles bovine insulin with the exception of one extra amino acid, methionine [40]. Sometimes referred to as "plant insulin", polypeptide-p tests negative in immunoassays against bovine insulin and reportedly does not invoke an antigenic response in humans. Clinical research examining the effect of polypeptide-p on blood sugar levels has mainly focused on the subcutaneous administration of this compound. By circumventing the neutralizing proteolytic enzymes of the intestines, polypeptide-p is believed to have greater activity when injected subcutaneously compared with oral administration [13].

In a study of nine patients with diabetes mellitus (six juvenile, two asymtomatic, one maturity onset) ranging from age 16 to 52 years, subcutaneous injection of polypeptide-p resulted in a statistically significant drop in mean blood sugar levels (2-tailed, paired $t$-test, $p=0.05$ ) [34]. No appreciable decrease in blood sugar levels was observed among either normal $(n=5)$ or diabetic $(n=5)$ referents receiving placebo injections. However, participants were not randomized between bitter melon and placebo. Compared with a typical peak effect seen after 2-3 h for regular insulin, polypeptide-p treated patients peaked after 4-12 h. A similar hypoglycemic effect was observed in a subsequent (non-randomized) study (presented at the 20th Annual Meeting of the American Society of Pharmacognosy, Purdue University) in which polypeptide-p was subcutaneously injected into 11 diabetic patients (five juvenile, six maturity onset) compared with eight diabetic referents (six juvenile, two maturity onset) [22]. Those with a history of ketoacidosis, cerebrovascular accidents, acute myocardial infarction, and renal failure were excluded from the study.

Among non-insulin-dependent diabetics, a water-soluble extract from locally obtained fresh (raw) bitter melon juice has been observed to significantly reduce mean blood sugar levels and correspondingly increase mean insulin levels during a $50 \mathrm{~g}$ oral glucose test, compared with standard test of distilled water $(p<0.05)$ [42]. Frozen juice squeezed from bitter melon pulp and seeds from the plant manifested equally positive results in studies of diabetics $[37,50]$. However, a weaker effect upon glucose levels was reported following the consumption of either fried fruit or dried powder forms of bitter melon [33,42]. A tea prepared from bitter melon leaves reduced HbA1c levels by $63 \%$ ( $p=0.005)$ compared with a referent tea (Camellia Sinensis) [46]. Anecdotally, some of the patients who received bitter melon powder for 7 days claimed to remain well and complication-free without any medication for about 1-2 months [33]. In a preliminary open-label uncontrolled supplementation trial of 42 participants, $4.8 \mathrm{~g}$ of lyophilized wild bitter melon powder significantly decreased the rate of metabolic syndrome after three months of supplementation and the improved status remained for one month after the supplementation ceased $(p=0.021)$ [49]. An increase of insulin resistance also was noted for visit 2 , but did not reach statistical significance. In a small case series of seven patients, an aqueous extract of $100 \mathrm{~g}$ of chopped bitter melon fruit taken each morning for 3 weeks resulted in a 
$54 \%$ drop in blood sugar levels $(p<0.01)$ [47]. The effect was much lower $(25 \%$ drop $)$ in the powder-treated group. Furthermore, drinking an aqueous suspension of bitter melon pulp led to decreased levels of both fasting and post-prandial blood sugar (mean reduction of $18 \%$ ) in a case series of 100 moderate non-insulin dependent diabetics [32].

A statistically significant decrease $(p<0.001)$ in post-prandial blood sugar level was observed among patients administered $4 \mathrm{~g}$ of bitter melon fruit powder for 21 days in a cross-over study of 8 non-insulin dependent diabetic patients (five males, three females already taking $1 / 2-1$ tablets daily of glibenclamide) randomly selected from the diabetic center in Rajshahi, Bangladesh [38]. Patients ranged in age from 35 to 60 years. Each was served the same breakfast items before a $5 \mathrm{~mL}$ blood sample was taken $2 \mathrm{~h}$ later. A combination regimen of bitter melon and fenugreek seeds was found to have a synergistic effect in lowering blood sugar levels. In a similar study, a powdered mixture of bitter melon fruit, jamun seeds, and fenugreek seeks administered to 60 non-insulin-dependent male diabetics significantly reduced their fasting and postprandial glucose levels and decreased their dose of oral hypoglycemic drugs [41].

Only a few randomized controlled trials of bitter melon have been conducted. Study designs differed and a meta-analysis pooling results was not performed. A statistically significant mean decrease in fructosamine, following a 4 -week regimen of $2,000 \mathrm{mg} /$ day of bitter melon, compared with baseline levels, was reported in a clinical trial (randomization to 4 groups, intention-to-treat design) of patients with newly diagnosed type 2 diabetes conducted at four hospitals in Thailand $(-10.2 ; 95 \%$ confidence interval=-19.1-1.3 $\mu \mathrm{mol} / \mathrm{L}$ ) [36]. Roasted rice powder (which has a high glycemic load) was used as the placebo comparison. Paradoxically, bitter melon had no apparent effect on fasting glucose or 2-hour glucose tolerance measurements. Three other groups were tested in this study and it is not clear if the statistical significance of the above finding holds after adjustment for multiplicity. In another positive randomized clinical study $(n=15$ noninsulin dependent diabetics), bitter melon was observed to act in synergy with two oral hypoglycemics (metformin, glibenclamide) [48]. After 7 days of treatment, greater than full dose efficacy ( $10 \%$ to $21 \%$ reduced sugar levels) was seen for bitter melon plus half doses of metformin and glibenclamide, illustrating the potentiated effect of the combined administration in diabetics. However, residual confounding, given the small sample of the study, could have biased the results.

No statistically significant change in serum fructosamine over time (baseline to 4 weeks of treatment) was observed for bitter melon $(6,000 \mathrm{mg}$ /day) compared with placebo (riboflavin) in a randomized controlled clinical trial (treatment $n=26$; Placebo $n=24$ ) [39]. While a mean drop in fructosamine from 350.8 to $319.1 \mathrm{mg} / \mathrm{dL}$ was observed for the bitter melon arm from baseline to 4 weeks, an unexplained drop in the placebo group also was observed over the same time period (349.1 to $333.9 \mathrm{mg} / \mathrm{dL}$ ). Details were not provided regarding the standardization of bitter melon or whether patients complied with their routine oral hypoglycemic agents. Similarly, the mean beneficial change in fasting blood sugar levels from pre- to post-treatment ( 3 months, mean decrease $=0.41 \mathrm{mmol} / \mathrm{L}$ ) with bitter melon was not statistically significant in a randomized, double-blind, placebo-controlled trial conducted at outpatient clinics in the Philippines [35]. However, the achieved power for this study was only $11 \%$. Another (non-peer reviewed) study, conducted over a 10-year period by the Philippine Council for Health Research and Development (PCHRD) reported that a 100-milligram per kilo dose per day of bitter melon was clinically comparable to $2.5 \mathrm{mg}$ of the anti-diabetic drug glibenclamide 
taken twice per day [45,53] for regulating blood sugar [45]. A pilot study conducted in five non-diabetic overweight men did not observe any effect on plasma glucose levels after they consumed a freeze-dried juice extract (100 mg/kg body weight) of bitter melon [14].

Recently, a prospective, randomized, double-blinded and placebo-controlled trial of bitter melon reported a statistically significance decrease in $\mathrm{HbA} 1 \mathrm{c}$ levels after 4 mouths of intervention, compared with a referent group receiving refined soybean oil [51]. In this study, bitter melon was administered as a special water soluble power ( $2 \mathrm{~g}$ daily dose), standardized at $10 \%$ charantin. Bitter melon was well-tolerated and no apparent differences in liver function values were observed in comparison with the referent group. In a study of 40 newly diagnosed patients with type 2 diabetes mellitus (18 males, 22 females), a single dose (100 mg/kg/day) of bitter melon was observed to be significantly more effective than those randomized to placebo (double-blind) in reducing mealtime glycemic excursions (within post-dose window) and reverting more rapidly to baseline levels [43].

Alone, few side effects have been associated with the use of bitter melon. No deaths have been reported to date. The most commonly observed adverse effects include mild diarrhea and abdominal pain, which subside after discontinuing use [54]. However, bitter melon has been shown to potentiate the effect of certain drugs used to treat diabetes, possibly resulting in hypoglycemia [52]. Rare cases of hypoglycemic coma and convulsions have been reported in children drinking bitter melon tea [55]. A single case report has suggested that bitter melon may induce paroxysmal atrial fibrillation, based on a Naranjo adverse drug reaction score of 6 (corresponding to a probable causal association) [56]. Bitter melon use also is contraindicated during pregnancy because of its abortifacient properties [57]. The risk associated with the long-term use of bitter melon has not been studied.

\section{Synthesis of the Literature}

Differences in the effectiveness observed across studies examining the potential glucose lowering effects of bitter melon may be attributable to several factors. Ayurvedic physicians typically insist on the daily preparation of herbal diabetic remedies intended for oral consumption [50]. The aqueous extract of Aegle Marmelos, a herb reputed to have oral hypoglycemic activity similar to bitter melon, reportedly loses $66 \%$ of its maximum hypoglycemic potency after just $72 \mathrm{~h}$ of room temperature storage [50]. The decline in potency is believed to be due to either bacterial activity or other yet-to-be understood explanations [50]. Factors potentially influencing bitter melon efficacy include differences in soil/climate, harvest time, part of the plant used (e.g., leaves are believed to be more effective), and how the plant is cooked and prepared (e.g., over-heating, the addition of other ingredients that may affect absorption). Different variants of bitter melon (shape, color, size) also may be found throughout the world and could affect the biologic activity of the herb [58]. Information on the above factors typically was not collected in the studies considered for this review. Furthermore, studies often lacked a population-based referent group, were not adequately blinded, failed to conceal allocation assignment, were too short in duration to yield effect, or suffered from selection bias (not randomized) [36]. Such biases tend to be associated with inflated study benefits $[59,60]$. In other cases, statistical methodology, design details, and study conduct (e.g., inclusion/exclusion criteria, ethnic background of patients, drop-out rates, a priori power analysis, baseline comparability, outcome measures, and treatment adherence/integrity) were not adequately described to allow a definitive comparison across studies or a 
basic assessment of face validity [61,62]. Among the manuscripts reviewed in Table 2, less than $20 \%$ received a quality score of two or greater on the Jadad scale [62].

While bitter melon holds promise for the safe and affordable prevention and control of high blood sugar among pre-diabetics, primary care providers must err on the side of caution and appropriately advise patients not to forego medical consultation and conventional therapy (especially as their disease progresses) in lieu of anecdotal evidence or recommendation from traditional herbal practitioners. Patients with poor glycemic control, cardiovascular disease, fatty liver disease, and polycystic ovary syndrome must be closely monitored and queried regarding their use of bitter melon and other herbal products that may interact with conventional medicines such as metformin and acarbose [63,64]. Furthermore, drugs approved by the Food and Drug Administration (FDA) that increase insulin sensitivity (e.g., pioglitazone) may help reduce the risk of converting from pre-diabetes to diabetes, without the uncertainty in dosing and potential for interaction associated with the uncontrolled use of bitter melon. Bariatric surgery also remains a proven intervention to control the progression to diabetes [2].

Nevertheless, the use of bitter melon may be warranted in certain individuals, such as patients without health insurance, those living in rural areas far from health care, members of ethnic/religious groups who do not subscribe to conventional medical care or surgery, and non-compliant patients (e.g., those with difficulty swallowing pills). An increasing prevalence of pre-diabetics has been noted in remote, rural regions, especially those with a high number of elderly residents [65]. Further assessment of the benefits and risks of bitter melon use in these groups is recommended.

Reducing healthcare costs also may be a factor prompting the use of bitter melon and other alternative therapies aimed at preventing and controlling high blood sugar. An advantage of medicinal plants such as bitter melon is that they may be inexpensively cultivated in gardens and greenhouses. However, potential short-term savings must be weighed in light of the effectiveness of bitter melon (or lack thereof) and the proclivity of patients to delay using clinically proven therapies. Additionally, the acceptance of bitter melon may be limited due to its undesirable bitter taste [66]. Research needs to be directed toward strategies for improving the palatability and consumption intentions of food dishes containing bitter melon.

\section{Conclusions}

Overall, it remains controversial whether bitter melon has proven benefits in lowering blood sugar among pre-diabetics or aids in slowing the progression to diabetes. While the evidence to date, when examined as a whole, is suggestive of a possible beneficial effect, future clinical studies that meet rigorous methodological standards are warranted before attempts to establish policy recommendations regarding the use of this herb among pre-diabetics.

\section{Acknowledgments}

The authors thank the East Carolina Center for Health Disparities, East Carolina Heart Institute (ECHI) and the East Carolina Diabetes and Obesity Institute (ECDOI) for the resources to conduct this review. 


\section{Author Contributions}

Jimmy Efird and Yuk Ming Choi conceived and wrote the first draft and contributed to the critical revision of the manuscript. Stephen Davies, Sanjay Mehra, Ethan Anderson, and Lalage Katunga contributed to the writing, and critical revision of the manuscript. All authors approved the final version of the manuscript.

\section{Conflicts of Interest}

The authors declare no conflict of interest.

\section{References}

1. Center for Disease Control and Prevention (CDC). National Diabetes Fact Sheet. 2011. Avaialable online: www.cdc.gov/diabetes/pubs/pdf/ndfs_2011.pdf (accessed on 14 December 2013).

2. Garber, A.J.; Abrahamson, M.J.; Barzilay, J.I.; Blonde, L.; Bloomgarden, Z.T.; Bush, M.A.; Dagogo-Jack, S.; Davidson, M.B.; Einhorn, D.; Garvey, W.T.; et al. American Association of Clinical Endocrinologists' comprehensive diabetes management algorithm 2013 consensus statement-Executive summary. Endocr. Pract. 2013, 19, 536-557.

3. Shah, A.S.; Gao, Z.; Urbina, E.M.; Kimball, T.R.; Dolan, L.M. Pre-diabetes: The effects on arterial thickness and stiffness in obese youth. J. Clin. Endocrinol. Metab. 2014, doi:10.1210/jc.2013-3519.

4. Milman, S.; Crandall, J.P. Mechanisms of vascular complications in prediabetes. Med. Clin. North Am. 2011, 95, 309-325.

5. Handelsman, Y.; Mechanick, J.I.; Blonde, L.; Grunberger, G.; Bloomgarden, Z.T.; Bray, G.A.; Dagogo-Jack, S.; Davidson, J.A.; Einhorn, D.; Ganda, O.; et al. AACE task force for developing diabetes comprehensive care plan. American Association of Clinical Endocrinologists medical guidelines for clinical practice for developing a diabetes mellitus comprehensive care plan. Endocr. Pract. 2011, 17(Suppl 2), 1-53.

6. National Center for Complementary and Alternative Medicine (NCCAM). Complementary, Alternative, or Integrative Health: What's in a Name? U.S. Department of Health and Human Services, National Institutes of Health: Bethesda, MD, USA. Available online: http://nccam.nih.gov/health/whatiscam (accessed on 6 November 2013).

7. Dham, S.; Shah, V.; Hirsch, S.; Banerji, M.A. The role of complementary and alternative medicine in diabetes. Curr. Diab Rep. 2006, 6, 251-258.

8. Abel, W.M.; Efird, J.T. The association between trust in health care providers and medication adherence among Black women with hypertension. Front. Public. Health. 2013, 1, doi:10.3389/fpubh.2013.00066.

9. Gurib-Fakim, A. Medicinal plants: Traditions of yesterday and drugs of tomorrow. Mol. Aspects Med. 2006, 27, 1-93. 
10. Mahomoodally, M.F.; Subratty, A.H.; Gurib-Fakim, A.; Choudhary, M.I.; Nahar Khan, S. Traditional medicinal herbs and food plants have the potential to inhibit key carbohydrate hydrolyzing enzymes in vitro and reduce postprandial blood glucose peaks. Sci. World J. 2012, doi:10.1100/2012/285284.

11. Yin, J.; Zhang, H.; Ye, J. Traditional Chinese medicine in treatment of metabolic syndrome. Endocr. Metab. Immune Disord. Drug Targets 2008, 8, 99-111.

12. Rizvi, S.I.; Mishra, N. Traditional Indian medicines used for the management of diabetes mellitus. J. Diabetes Res. 2013, doi:10.1155/2013/712092.

13. Raman, A.; Lau, C. Anti-diabetic properties and phytochemistry of Momordica charantia I. (Cucurbitaceae). Phytomedicine 1996, 2, 349-362.

14. Kasbia, G.S.; Arnason, J.T.; Imbeault, P. No effect of acute, single dose oral administration of Momordica charantia Linn., on glycemia, energy expenditure and appetite: A pilot study in non-diabetic overweight men. J. Ethnopharmacol. 2009, 126, 127-133.

15. Singh, J.; Cumming, E.; Manoharan, G.; Kalasz, H.; Adeghate, E. Medicinal chemistry of the anti-diabetic effects of Momordica charantia: Active constituents and modes of actions. Open Med. Chem. J. 2011, 5, 70-77.

16. Hazarika, R.; Parida, P.; Neog, B.; Yadav, R.N. Binding energy calculation of GSK-3 protein of human against some anti-diabetic compounds of Momordica charantia Linn. (Bitter Melon). Bioinformation 2012, 8, 251-254.

17. Tan, M.J.; Ye, J.M.; Turner, N.; Hohnen-Behrens, C.; Ke, C.Q.; Tang, C.P.; Chen, T.; Weiss, H.C.; Gesing, E.R.; Rowland, A.; et al. Antidiabetic activities of triterpenoids isolated from bitter melon associated with activation of the AMPK pathway. Chem. Biol. 2008, 15, 263-273.

18. Klomann, S.D.; Mueller, A.S.; Pallauf, J.; Krawinkel, M.B. Antidiabetic effects of bitter gourd extracts in insulin-resistant $d b / d b$ mice. Br. J. Nutr. 2010, 104, 1613-1620.

19. Chhabra, G.; Dixit, A. Structure modeling and antidiabetic activity of a seed protein of Momordica charantia in non-obese diabetic (nod) mice. Bioinformation 2013, 9, 766-770.

20. Nerurkar, P.V.; Lee, Y.K.; Motosue, M.; Adeli, K.; Nerurkar, V.R. Momordica charantia (Bitter Melon) reduces plasma apolipoprotein B-100 and increases hepatic insulin receptor substrate and phosphoinositide-3 kinase interactions. Br. J. Nutr. 2008, 100, 751-759.

21. Wang, Z.Q.; Zhang, X.H.; Yu, Y.; Poulev, A.; Ribnicky, D.; Floyd, Z.E.; Cefalu, W.T. Bioactives from bitter melon enhance insulin signaling and modulate acyl carnitine content in skeletal muscle in high-fat diet-fed mice. J. Nutr. Biochem. 2011, 22, 1064-1073.

22. Meir, P.; Yaniv, Z. An in vitro study on the effect of Momordica charantia on glucose uptake and glucose metabolism in rats. Planta Med. 1986, 51, 12-16.

23. Shibib, B.A.; Khan, L.A.; Rahman, R. Hypoglycaemic activity of Coccinia indica and Momordica charantia in diabetic rats: Depression of the hepatic gluconeogenic enzymes glucose-6-phosphatase and fructose-1,6-bisphosphatase and elevation of both liver and red-cell shunt enzyme glucose-6-phosphate dehydrogenase. Biochem. J. 1993, 292, 267-270.

24. Shih, C.C.; Lin, C.H.; Lin, W.L. Effects of Momordica charantia on insulin resistance and visceral obesity in mice on high-fat diet. Diabetes Res. Clin. Pract. 2008, 81, 134-143. 
25. Hafizur, R.M.; Kabir, N.; Chishti, S. Modulation of pancreatic beta-cells in neonatally streptozotocin-induced type 2 diabetic rats by the ethanolic extract of Momordica charantia fruit pulp. Nat. Prod. Res. 2011, 25, 353-367.

26. Singh, N.; Gupta, M. Regeneration of Beta cells in islets of langerhans of pancreas of alloxan diabetic rats by acetone extract of Momordica charantia (Linn.) (Bitter Gourd) fruits. Indian. J. Exp. Biol. 2007, 45, 1055-1062.

27. Chen, P.H.; Chen, G.C.; Yang, M.F.; Hsieh, C.H.; Chuang, S.H.; Yang, H.L.; Kuo, Y.H.; Chyuan, J.H.; Chao, P.M. Bitter melon seed oil-attenuated body fat accumulation in diet-induced obese mice is associated with cAMP-dependent protein kinase activation and cell death in white adipose tissue. J. Nutr. 2012, 142, 1197-1204.

28. Bao, B.; Chen, Y.G.; Zhang, L.; Na Xu Y.L.; Wang, X.; Liu, J.; Qu, W. Momordica charantia (Bitter Melon) reduces obesity-associated macrophage and mast cell infiltration as well as inflammatory cytokine expression in adipose tissues. PLoS One 2013, 8, doi:10.1371/journal.pone.0084075.

29. Chen, Q.; Li, E.T. Reduced adiposity in bitter melon (Momordica charantia) fed rats is associated with lower tissue triglyceride and higher plasma catecholamines. Br. J. Nutr. 2005, 93, 747-754.

30. Nerurkar, P.V.; Pearson, L.; Efird, J.T.; Adeli, K.; Theriault, A.G.; Nerurkar, V.R. Microsomal triglyceride transfer protein gene expression and $\mathrm{ApoB}$ secretion are inhibited by bitter melon in Hepg2 cells. J. Nutr. 2005, 135, 702-706.

31. Sasa, M.; Inoue, I.; Shinoda, Y.; Takahashi, S.; Seo, M.; Komoda, T.; Awata, T.; Katayama, S. Activating effect of momordin extract of bitter melon (Momordica charantia L.), on the promoter of human PPAR Delta. J. Atheroscler. Thromb. 2009, 16, 888-892.

32. Ahmad, N.; Hassan, M.R.; Halder, H.; Bennoor, K.S. Effect of Momordica charantia (Karolla) extracts on fasting and postprandial serum glucose levels in NIDDM patients. Bangladesh Med. Res. Counc. Bull. 1999, 25, 11-13.

33. Akhtar, M.S. Trial of Momordica charantia Linn. (Karela) powder in patients with maturity-onset diabetes. J. Pak. Med. Assoc. 1982, 32, 106-107.

34. Baldwa, V.S.; Bhandari, C.M.; Pangaria, A.; Goyal, R.K. Clinical trial in patients with diabetes mellitus of an insulin-like compound obtained from plant source. UPS J. Med. Sci. 1977, 82, $39-41$.

35. Dans, A.M.; Villarruz, M.V.; Jimeno, C.A.; Javelosa, M.A.; Chua, J.; Bautista, R.; Velez, G.G. The effect of Momordica charantia capsule preparation on glycemic control in type 2 diabetes mellitus needs further studies. J. Clin. Epidemiol. 2007, 60, 554-559.

36. Fuangchan, A.; Sonthisombat, P.; Seubnukarn, T.; Chanouan, R.; Chotchaisuwat, P.; Sirigulsatien, V.; Ingkaninan, K.; Plianbangchang, P.; Haines, S.T. Hypoglycemic effect of bitter melon compared with metformin in newly diagnosed type 2 diabetes patients. J. Ethnopharmacol. 2011, 134, 422-428.

37. Grover, J.; Gupta, S. Hypoglycemic activity of seeds of Momordica Charantia. Eur. J. Pharmacol. 1990, 183, 1026-1027.

38. Habib, A.; Gafur, A. The hypoglycaemic activity of karela fruits and fenugreek seeds in non-insulin dependent diabetic patients. Pakistan J. Pharm. 2003, 20, 37-40. 
39. John, A.J.; Cherian, R.; Subhash, H.S.; Cherian, A.M. Evaluation of the efficacy of bitter gourd (Momordica charantia) as an oral hypoglycemic agent-A randomized controlled clinical trial. Indian J. Physiol. Pharmacol. 2003, 47, 363-365.

40. Khanna, P.; Jain, S.C.; Panagariya, A.; Dixit, V.P. Hypoglycemic activity of polypeptide-P from a plant source. J. Nat. Prod. 1981, 44, 648-655.

41. Kochhar, A.; Nagi, M. Effect of supplementation of traditional medicinal plants on blood glucose in non-insulin-dependent diabetics: A pilot study. J. Med. Food 2005, 8, 545-549.

42. Leatherdale, B.A.; Panesar, R.K.; Singh, G.; Atkins, T.W.; Bailey, C.J.; Bignell, A.H. Improvement in glucose tolerance due to Momordica charantia (Karela). Br. Med. J. (Clin. Res. Ed) 1981, 282, 1823-1824.

43. Lim, S.T.; Jimeno, C.A.; Razon-Gonzales, E.B.; Velasquez, E.N. The MOCHA DM study: The effect of Momordica charantia tablets on glucose and insulin levels during the postprandial state among patients with type 2 diabetes mellitus. Phil. J. Inter. Med. 2010, 48, 19-25.

44. Pons, J.; Stevenson, D. The effects of Momordica charantia L. ("Cundeamor") in diabetes mellitus. Puert Rico J. Public Health Trop. Med. 1943, 19, 196-215.

45. Phillippine Council for Health Research and Development. DOH Reinstates Ampalaya as Diabetes Cure. Available online: http://asianjournalusa.com/doh-reinstates-ampalaya-as-diabetescure-p2449-59.htm (accessed on 27 October 2013).

46. Rosales, R.; Fernando, R. An inquiry to the hypoglycemic action of Momordica charantia among type 2 diabetic patients. Phil. J. Inter. Med. 2001, 39, 213-216.

47. Srivastava, Y.; Venkatakrishna-Bhatt, H.; Verma, Y.; Venkaiah, K.; Raval, B. Antidiabetic and adaptogenic properties of Momordica charantia extract: An experimental and clinical evaluation. Phytother. Res. 1993, 7, 285-289.

48. Tongia, A.; Tongia, S.K.; Dave, M. Phytochemical determination and extraction of Momordica charantia fruit and its hypoglycemic potentiation of oral hypoglycemic drugs in diabetes mellitus (NIDDM). Indian J. Physiol. Pharmacol. 2004, 48, 241-244.

49. Tsai, C.H.; Chen, E.C.; Tsay, H.S.; Huang, C.J. Wild bitter gourd improves metabolic syndrome: A preliminary dietary supplementation trial. Nutr. J. 2012, 11, doi:10.1186/1475-2891-11-4.

50. Welihinda, J.; Karunanayake, E.H.; Sheriff, M.H.; Jayasinghe, K.S. Effect of Momordica charantia on the glucose tolerance in maturity onset diabetes. J. Ethnopharmacol. 1986, 17, 277-282.

51. Zanker, K.S.; Mang, B.; Wolters, M.; Hahn, A. Personalized diabetes and cancer medicine: A rationale for anti-diabetic nutrition (bitter melon) in a supportive setting. Curr. Cancer Ther. Rev. 2012, 8, 66-77.

52. Aslam, M.; Stockley, I.H. Interaction between curry ingredient (Karela) and drug (Chlorpropamide). Lancet 1979, 1, 607.

53. Duque, F. Reinstating Ampalaya (Momordica charantia, L.) as Scientifically-Validated Herbal Medicinal Plant; Department of Health, Republic of the Philippines: Manila, Philippines, 2007.

54. Ooi, C.P.; Yassin, Z.; Hamid, T.A. Momordica charantia for type 2 diabetes mellitus. Cochrane Database Syst. Rev. 2010, doi:10.1002/14651858.CD007845.pub2.

55. Basch, E.; Gabardi, S.; Ulbricht, C. Bitter melon (Momordica charantia): A review of efficacy and safety. Am. J. Health. Syst. Pharm. 2003, 60, 356-359. 
56. Erden, I.; Ordu, S.; Erden, E.C.; Caglar, S.O. A case of atrial fibrillation due to Momordica charantia (bitter melon). Ann. Saudi Med. 2010, 30, 86-87.

57. Aguwa, C.N.; Mittal, G.C. Abortifacient effects of the roots of Momordica angustisepala. J. Ethnopharmacol. 1983, 7, 169-173.

58. Mahmood, A.; Raja, G.; Mahmood, T.; Gulfraz, M.; Khanum, A. Isolation and characterization of antimicrobial activity conferring component(s) from seeds of bitter gourd (Momordica charantia). J. Med. Plants Res. 2013, 6, 566-573.

59. Schulz, K.F.; Chalmers, I.; Hayes, R.J.; Altman, D.G. Empirical evidence of bias-Dimensions of methodological quality associated with estimates of treatment effects in controlled trials. JAMA 1995, 273, 408-412.

60. Colditz, G.A.; Miller, J.N.; Mosteller, F. How study design affects outcomes in comparisons of therapy. I: Medical. Stat. Med. 1989, 8, 441-454.

61. DerSimonian, R.; Charette, L.J.; McPeek, B.; Mosteller, F. Reporting on methods in clinical trials. N. Engl. J. Med. 1982, 306, 1332-1337.

62. Jadad, A.R.; Moore, R.A.; Carroll, D.; Jenkinson, C.; Reynolds, D.J.; Gavaghan, D.J.; McQuay, H.J. Assessing the quality of reports of randomized clinical trials: Is blinding necessary? Control. Clin. Trials 1996, 17, 1-12.

63. Lord, J.M.; Flight, I.H.; Norman, R.J. Metformin in polycystic ovary syndrome: Systematic review and meta-analysis. BMJ 2003, 327, 951-953.

64. Abbasi, F.; Chu, J.W.; McLaughlin, T.; Lamendola, C.; Leary, E.T.; Reaven, G.M. Effect of metformin treatment on multiple cardiovascular disease risk factors in patients with type 2 diabetes mellitus. Metabolism 2004, 53, 159-164.

65. Misra, P.; Upadhyay, R.P.; Misra, A.; Anand, K.A. Review of the epidemiology of diabetes in rural India. Diabetes Res. Clin. Pract. 2011, 92, 303-311.

66. Snee, L.S.; Nerurkar, V.R.; Dooley, D.A.; Efird, J.T.; Shovic, A.C.; Nerurkar, P.V. Strategies to improve palatability and increase consumption intentions for Momordica charantia (bitter melon): A vegetable commonly used for diabetes management. Nutr. J. 2011, 10, doi:10.1186/1475-2891-10-78.

(C) 2014 by the authors; licensee MDPI, Basel, Switzerland. This article is an open access article distributed under the terms and conditions of the Creative Commons Attribution license (http://creativecommons.org/licenses/by/3.0/). 